\title{
MECHANISMS OF DE - AND REMYELINATION IN AUTOIMMUNE ENCEPHALOMYELITIS AND MULTIPLE SCLEROSIS
}

\author{
Hans Lassmann, ${ }^{1,2}$ Gerda Suchanek, ${ }^{1}$ and Mascha Schmied ${ }^{1}$ \\ ${ }^{1}$ Research Unit for Experimental Neuropathology, Austrian Academy of Sciences \\ ${ }^{2}$ Neurological Institute, University Vienna, Austria
}

\section{INTRODUCTION}

Several different immunopathological mechanisms have been suggested to be involved in the destruction of myelin in inflammatory demyelinating diseases. They include destruction of oligodendrocytes by virus infection ${ }^{1}$ as well as immune mediated damage by macrophage products ${ }^{2}$, specific antibodies ${ }^{3}$ or T-lymphocytes ${ }^{4}$. To evaluate the validity of these concepts it is of critical importance to determine the fate of oligodendrocytes within the lesions $5,6,7,8$. This, however, was difficult and inconclusive because of the lack of specific and sensitive markers that allow the identification of oligodendrocytes in demyelinated plaques. Recently, new techniques became available to address this question, which have been applied in the present study.

\section{MATERIALS AND METHODS}

Our study was performed on three different inflammatory demyelinating diseases. Subacute demyelinating encephalomyelitis was induced in rats by intracerebral infection with corona virus ${ }^{9}$. The model of chronic relapsing autoimmune encephalomyelitis was established by repeated passive cotransfer of encephalitogenic T-lymphocytes directed against myelin basic protein with demyelinating monoclonal antibodies against myelin oligodendroglia glycoprotein ${ }^{10}$. In addition inflammatory demyelinating plaques were studied in biopsy and autopsy material from 25 cases of multiple sclerosis with a disease duration ranging from few weeks to several years.

Appropriate lesions were selected from a large number of sections and screened by routine neuropathology. Immunocytochemistry was performed with a biotin avidin technique ${ }^{11}$ with monoclonal and polyclonal antibodies against the following myelin antigens: MOG (818C5, Y1 and Y12; a gift from Dr. S. Piddlesden, University Cardiff, UK), CNPase (Affinity 
Research Products, Ilkeston, UK), MBP (a gift from Dr. J.M. Matthieu, University Lausanne, Switzerland), PLP (a gift from Dr. S. Piddlesden, University Cardiff, UK) and HNK1 (Leu7, Becton and Dickinson, Vienna, Austria). The protocol for in situ hybridization with specific probes for MBP, PLP, CNPase, MAG and MOG has been described in detail before ${ }^{12}$. A method for in situ nick translation was developed for the detection of DNA strand breaks in tissue sections, which allows the identification of degenerating cells within the lesions ${ }^{13}$.

\section{RESULTS AND DISCUSSION}

\section{Detection of oligodendrocytes in normal adult and developing rat central nervous system tissue}

As described in detail earlier oligodendrocytes can be detected in adult and developing brain tissue by immunocytochemistry for MOG and CNPase. MOG is expressed on the surface of oligodendrocytes and myelin sheaths, whereas CNPase is located in the cytoplasm of oligodendrocytes and their processes ${ }^{14}$. The mRNAs for MBP, PLP, MAG and CNPase were found in the cytoplasm of oligodendrocytes during active myelination (Figure 1a,b) and also in adult animals ${ }^{12}$. MOG mRNA appeared later during the process of myelination, but was prominently detectable in a subpopulation of oligodendrocytes in adult rats. Furthermore, MOG is expressed on the surface of oligodendrocytes, that survive in demyelinated lesions, such as those found during Wallerian degeneration ${ }^{15}$.

\section{Oligodendrocytes in corona virus induced subacute demyelinating encephalomyelitis}

In this model during the acute phase of demyelination numerous oligodendrocytes could be identified, which were double labelled by in situ nick translation and immunocytochemistry for MOG and CNPase. Only a small fraction of these degenerating oligodendrocytes also contained detectable virus antigen by immunocytochemistry. This suggests that also uninfected oligodendrocytes can be destroyed within the lesions in the course of the immune reaction. In more chronic plaques, no oligodendrocytes were detected by either immuno-cytochemistry for MOG or CNPase or by in situ hybridization for PLP mRNA in the center of the lesions (Figure 1c,d). Increased mRNA for myelin proteins was detected at the edge of the lesions, indicating central remyelination from the lesional borders.

Oligodendrocytes in chronic relapsing autoimmune encephalomyelitis

As described before, cotransfer of MBP reactive T-lymphocytes with demyelinating antibodies leads to the formation of confluent demyelinated lesions in the central nervous system $^{3}$.

Furthermore, repeated cotransfers resulted in chronic relapsing clinical disease and persistently demyelinated lesions with little remyelination ${ }^{10}$. In the early stages of such chronic lesions, some cells, which showed DNA fragmentation by in situ nick translation, were labelled by anti-MOG and anti-CNPase antibodies. Furthermore, even 2 days after the last transfer, only very few cells, expressing oligodendroglia markers by immunocytochemistry or in situ hybridization were found in the areas of demyelination. In 

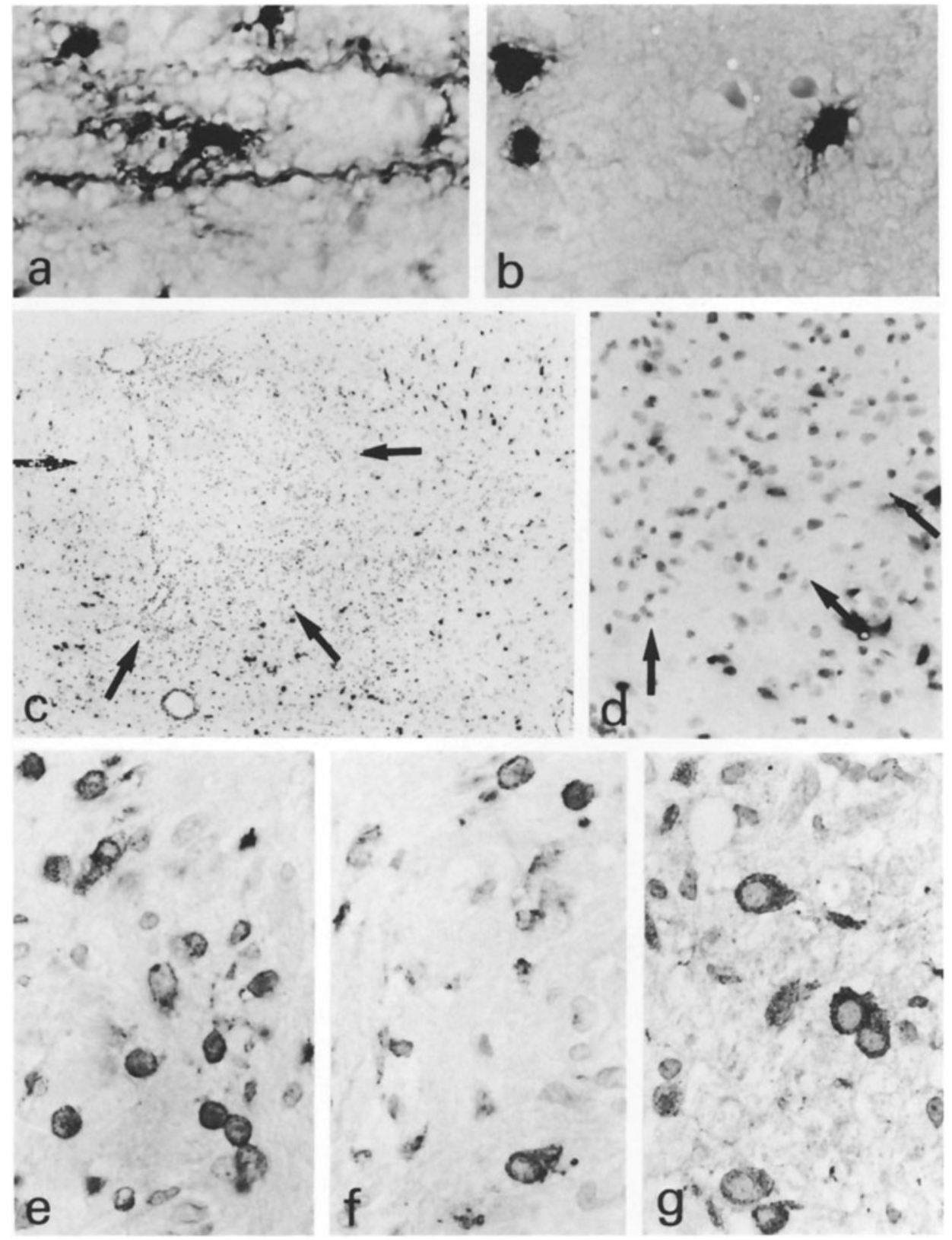

Figure 1. Oligodendrocytes in normal and diseased central nervous system tissue. a.) 7 day old rat, normal brain; in situ hybridization for MBPmRNA labels oligodendrocyte cell bodies and processes. $x 750 \mathrm{~b}$.) 7 day old rat, normal brain; in situ hybrydization for PLPmRNA; only the perinuclear cytoplasm is stained. $x 750 \mathrm{c}$.) Adult rat with corona virus induced subacute demyelinating encephalomyelitis. A demyelinated plaque (arrows) contains no oligodendrocytes. Numerous oligodendrocytes are labelled in the periplaque white matter; in situ hybridization for PLPmRNA; $x 75$ d.) Higher magnifications of figure 1c, showing the plaque border (arrows); in situ hybridization for PLPmRNA; $x 300 e$.) Inactive multiple sclerosis plaque; immuno-cytochemistry with antiMOG antibodies shows numerous small (inactive) oligodendrocytes. $x 750 \mathrm{f}$.) Inactive multiple sclerosis plaque; 2 large, activated oligodendrocytes were labelled by immunocytochemistry for MOG. g.) Inactive multiple sclerosis plaque; immunocytochemistry with anti-CNPase antibodies mainly labels large activated oligodendrocytes. x750 
the periphery of the lesions, however, an increased number of cells were identified that expressed mRNAs for myelin proteins. Thus, in chronic cotransfer autoimmune encephalomyelitis oligodendrocytes are destroyed during active demyelination. Remyelination is limited and occurs at the periphery of the plaques.

\section{Oligodendrocytes in multiple sclerosis lesions}

Since most of the multiple sclerosis brain tissue available came from autopsy material, our studies mainly involved immunocytochemistry for MOG and CNPase (figure 1e,f,g). Both antigens are readily detectable in paraffin embedded autopsy tissue. Within the demyelinated lesions of multiple sclerosis the density of immunocytochemically detectable oligodendrocytes was very variable, ranging within plaques from different patients from complete absence to similar numbers as compared to the adjacent white matter. Interestingly, within a single patient the number of oligodendrocytes per area was fairly constant between different plaques and showed little variation in relation to the stage of demyelinating activity.

Regarding oligodendroglia pathology in multiple sclerosis three different patterns were observed. In the vast majority of typical chronic multiple sclerosis cases, in patients with a disease duration of more than 3 years, the plaques revealed extensive loss of oligodendrocytes. On the contrary, in the majority of plaques, derived from patients at the first or second attack of the disease, oligodendrocytes were present in nearly normal numbers, regardless of the stage of demyelinating activity. In patients with Marburg's type ${ }^{16}$ of acute multiple sclerosis a pronounced loss of oligodendrocytes was found together with extensive destruction of other elements of the nervous system such as axons or astrocytes.

\section{Conclusions}

Our studies revealed that massive destruction and loss of oligodendrocytes occurs in chronic models of virus induced and autoimmune mediated demyelinating encephalomyelitis. Since oligodendrocytes are also destroyed in acute demyelinating encephalomyelitis, induced by a single cotransfer of encephalitogenic T-cells and demyelinating antibodies, the impairment of remyelination may be due to progressive depletion of the pool of adult oligodendrocyte progenitor cells, capable of differentiating into mature remyelinating oligodendrocytes in a similar way as suggested for models of toxic demyelination ${ }^{17}$. The lesions formed in such experimental models closely resemble those found in typical chronic multiple sclerosis.

In addition, however, our studies indicate that in multiple sclerosis the mechanisms of demyelination may be different in the lesions formed during early exacerbations of the disease. In these plaques we found a pronounced preservation of oligodendrocytes throughout all stages of demyelinating activity. This suggests that during the first or second relapse, at least in a subgroup of multiple sclerosis patients, not the oligodendrocytes but the myelin itself is the primary target of the immune attack. Therefore it has to be considered that the immunopathogenesis of the lesions may differ in individual patients between early and late exacerbations in the course of their disease. 


\section{REFERENCES}

1. R. Johnson, Viral aspects of multiple sclerosis, in: "Handbook of Clinical Neurology. Demyelinating Diseases," J. C. Koetsier, ed., Elsevier Science Publishers, Amsterdam (1985).

2. C. Griot, T. Bürge, M. Vanvelde, E. Peterhans, Antibody induced generation of reactive oxygen radicals by brain macrophages in canine distemper encephalitis: a mechanism for bystander demyelination, Acta Neuropathol. 78:396 (1989).

3. C. Linington, M. Bradl, H. Lassmann, C. Brunner, K. Vass, Augmentation of demyelination in rat acute allergic encephalomyelitis by circulating mouse monoclonal antibodies directed against a myelin/oligodendrocyte glycoprotein. Amer. J. Pathol. 130:443 (1988).

4. K. Selmaj, C.F. Brosnan, C.S. Raine, Expression of heat shock protein-65 by oligodendrocytes in vivo and in vitro: implications for multiple sclerosis, Neurology 42:795 (1992).

5. C.S. Raine, Multiple sclerosis. Oligodendrocyte survival and proliferation in an active lesion, Lab. Invest. 45:534 (1981).

6. H. Lassmann. "Comparative Neuropathology of Chronic Experimental Allergic Encephalomyelitis and Multiple Sclerosis," Springer-Verlag, Berlin Heidelberg New York Tokyo (1983).

7. J.W. Prineas, The neuropathology of multiple sclerosis, in: "Handbook of Clinical Neurology. Demyelinating Diseases," J.C. Koetsier, ed., Elsevier Science Publishers, Amsterdam (1985).

8. J.W. Prineas, E.E. Kwon, P.Z. Goldenberg, A.A. Ilyas, R.H. Quarles, J.A. Benjamins, T.J. Sprinkle, Multiple sclerosis: oligodendrocyte proliferation and fresh lesions. Lab. Invest. 61:489 (1989).

9. F. Zimprich, J. Winter, H. Wege, H. Lassmann, Corona virus induced primary demyelination: indications for the involvement of humoral immune response, Neuropath. Appl. Neurobiol. 17:469 (1991).

10. C. Linington, B. Engelhardt, G. Kapocs, H. Lassmann, Induction of persistently demyelinated lesions in the rat following the repeated adoptive transfer of encephalitogenic $\mathrm{T}$ cells and demyelinating antibody, J. Neuroimmunol. 40:219 (1992).

11. K. Vass, H. Lassmann, H. Wekerle, H.M. Wisniewski, The distribution of Ia-antigen in the lesions of rat acute experimental allergic encephalomyelitis, Acta Neuropathol. 70:149 (1986).

12. H. Breitschopf, G. Suchanek, R.M. Gould, D.R. Colman, H. Lassmann, In situ hybridization with digoxigenin-labeled probes: sensitive and reliable detection method applied to myelinating rat brain. Acta Neuropathol. 84:581 (1992).

13. R. Gold, M. Schmied, G. Rothe, H. Zischler, H. Breitschopf, H. Wekerle, H. Lassmann, Detection of DNA fragmentation at the cellular level by in situ nick translation: application to in vitro systems and tissue sections. J. Histochem. Cytochem. 41:1023 (1993).

14. C. Brunner, H. Lassmann, Th. V. Waehnelt, J.M. Matthieu, C. Linington, Differential ultrastructural localization of myelin basic protein, myelin/oligodendroglia glycoprotein and 2'3'-cyclic nucleotide 3'phosphodiesterase in the CNS of adult rats. J. Neurochem. 52:296 (1989).

15. S.K. Ludwin, Oligodendrocyte survival in Wallerian degeneration, Acta Neuropath. 80:184 (1990).

16. 0. Marburg, Die sogenannte "akute Multiple Sklerose", Jahrb. Psychiatrie 27:211 (1906).

17. S.K. Ludwin, Chronic demyelination inhibits remyelination in the central nervous system. An analysis of contributing factors, Lab. Invest. 43:382 (1980). 